PROCEEDINGS OF THE

AMERICAN MATHEMATICAL SOCIETY

Volume 126, Number 10, October 1998, Pages 3075-3078

S 0002-9939(98)04630-9

\title{
UNIQUE DECOMPOSITION OF RIEMANNIAN MANIFOLDS
}

\author{
J.-H. ESCHENBURG AND E. HEINTZE \\ (Communicated by Christopher Croke)
}

\begin{abstract}
We prove an extension of de Rham's decomposition theorem to the non-simply connected case.
\end{abstract}

\section{INTRODUCTION}

A connected Riemannian manifold may allow more than one decomposition into a product of indecomposable factors: Euclidean space of dimension $\geq 2$ splits orthogonally into a product of one-dimensional subspaces in many different ways. But by the classical theorem of de Rham ([dR], cf. also $[\mathrm{KN}],[\mathrm{M}],[\mathrm{P}]$ ), this is essentially the only simply connected example with that property. The purpose of our note is to generalize this result to the non-simply connected case as well.

Theorem. Any complete connected Riemannian manifold $M$ decomposes into a Riemannian product

$$
M=M_{0} \times M_{1} \times \ldots \times M_{p}
$$

where $M_{0}$ is a maximal factor isometric to euclidean space and each $M_{i}, i>0$, is indecomposable. This decomposition is unique up to the order of $M_{1}, \ldots, M_{p}$.

We call a Riemannian manifold indecomposable if it is not isometric to a Riemannian product of lower dimensional manifolds. Any (holonomy) irreducible manifold is indecomposable and by de Rham's theorem also the converse is true for simply connected manifolds. But in general the two notions differ: A non-rectangular flat 2-torus is indecomposable but not irreducible. By decomposing a manifold further and further it is clear that any Riemannian manifold admits a decomposition into a product of indecomposable ones. Therefore, the only question is about uniqueness. We say that a product decomposition is unique if the corresponding foliations are uniquely determined.

If $M$ is compact, there is no euclidean factor. Hence we get the following

Corollary 1. Let $M$ be a compact Riemannian manifold. Then $M$ decomposes uniquely into a Riemannian product of indecomposable factors. Any isometry of $M$ must preserve or interchange these factors. In particular, for any Riemannian product decomposition $M=M_{1} \times M_{2}$, the identity component of the isometry group splits as $I_{0}(M)=I_{0}\left(M_{1}\right) \times I_{0}\left(M_{2}\right)$.

Received by the editors February 28, 1997.

1991 Mathematics Subject Classification. Primary 53C20; Secondary 53C12.

Key words and phrases. Riemannian products, indecomposable Riemannian manifolds, irreducible Riemannian manifolds, de Rham's theorem. 
Another immediate consequence is a theorem due to Uesu [U] generalizing a previous result of Takagi [T]:

Corallary 2. Let $M, N$ and $B$ be complete connected Riemannian manifolds. If $M \times B$ is isometric to $N \times B$ then $M$ is isometric to $N$.

The main idea of the proof of our Theorem is to use a special (so-called "short") set of generators of the fundamental group which is compatible to any Riemannian decomposition of $M$. The same generating set had been used by Gromov in order to estimate the number of generators of the fundamental group (cf. $[\mathrm{G}]$ ).

\section{Proof of The Theorem}

By the remark above, we only have to show uniqueness. This will follow from a series of lemmas. We always denote by $\tilde{M}$ the universal cover of $M$ and by $\Gamma$ its group of deck transformations. A decompositon of $\tilde{M}$ into a product $\tilde{M}=$ $X_{1} \times \ldots \times X_{k}$ determines $k$ foliations on $\tilde{M}$ whose leaves through a point $p \in \tilde{M}$ will be denoted by $X_{i}(p)$. We say that an isometry $\phi$ of $\tilde{M}$ acts only on $X_{i}$ (or trivially on all $X_{j}, j \neq i$ ) if

$$
\phi\left(x_{1}, \ldots, x_{i}, \ldots, x_{k}\right)=\left(x_{1}, \ldots, \phi_{i} x_{i}, \ldots, x_{k}\right)
$$

for some isometry $\phi_{i}$ of $X_{i}$. In the language of foliations this means that each leaf $X_{i}(p)$ is $\phi$-invariant, i.e. $\phi(p) \in X_{i}(p)$ for all $p \in \tilde{M}$.

Lemma 1. The maximal euclidean factor $M_{0}$ of $M$ is uniquely determined.

Proof. By de Rham's theorem $\tilde{M}$ splits uniquely into $E \times N$ where $E$ is euclidean and $N$ has no euclidean factor. Furthermore $\Gamma$ preserves this splitting, i.e. each $\gamma \in$ $\Gamma$ is of the form $\left(\gamma_{E}, \gamma_{N}\right)$ where $\gamma_{E}$ and $\gamma_{N}$ are isometries of $E$ and $N$, respectively. Now any euclidean factor of $M$ corresponds to a factor $E_{1}$ of $E$ on which $\Gamma$ acts trivially. If $E=E_{1} \times E_{2}$ as a Riemannian manifold, then $E_{i}(x)=\vec{E}_{i}+x$ where $\vec{E}_{1} \oplus \vec{E}_{2}$ is an orthogonal splitting of the euclidean vector space $\vec{E}$ acting simply transitively on the affine space $E$ by translations. By the remark before Lemma 1 , $\gamma \in \Gamma$ acts trivially on $E_{1}$ if

$$
\gamma_{E}(x) \in E_{2}(x)=\vec{E}_{2}+x
$$

for all $x \in E$ which in turn is equivalent to $\left(\gamma_{E} x-x\right) \perp \vec{E}_{1}$. Thus $E_{1}$ is maximal if

$$
\vec{E}_{1}=\left\{\gamma_{E} x-x ; x \in E, \gamma \in \Gamma\right\}^{\perp} \subset \vec{E}_{1}
$$

but this is uniquely determined.

Lemma 2. Let $\tilde{M}=\tilde{M}_{1} \times \ldots \times \tilde{M}_{p}=\tilde{M}_{1}^{\prime} \times \ldots \times \tilde{M}_{q}^{\prime}$ be two decompositions of $\tilde{M}$. Then there exists a decomposition $\tilde{M}=\prod_{i, j} \tilde{M}_{i j} \times F$ of $\tilde{M}$ where $F$ is a euclidean factor and $\tilde{M}_{i j}(p)=\tilde{M}_{i}(p) \cap \tilde{M}_{j}^{\prime}(p)$.

Proof. $\tilde{M}_{i}(p)$ and $\tilde{M}_{j}^{\prime}(p)$ are totally convex in the sense that any minimal geodesic of $\tilde{M}$ joining two points in $\tilde{M}_{i}(p)$ or $\tilde{M}_{j}^{\prime}(p)$ lies completely in $\tilde{M}_{i}(p)$ or $\tilde{M}_{j}^{\prime}(p)$, respectively. Therefore, $\tilde{M}_{i j}(p)$ is a totally geodesic connected submanifold of $\tilde{M}$. The tangent spaces $\mathcal{D}_{i j}(p)=T_{p}\left(\tilde{M}_{i j}(p)\right)=T_{p}\left(\tilde{M}_{i}\right) \cap T_{p}\left(\tilde{M}_{j}^{\prime}\right)$ form a distribution $\mathcal{D}_{i j}$ which is invariant under parallel translations. Therefore we get from de Rham's theorem $\tilde{M}=\prod_{i, j} \tilde{M}_{i j} \times F$ where $F$ is some complementary factor. Since each 
irreducible de Rham factor is contained in some $\tilde{M}_{i}$ and in some $\tilde{M}_{j}^{\prime}$, it is also contained in some $\tilde{M}_{i j}$. Thus the complementary factor $F$ must be euclidean.

Recall that a splitting $\tilde{M}=\tilde{M}_{1} \times \ldots \times \tilde{M}_{p}$ of the universal cover is induced by a splitting $M=M_{1} \times \ldots \times M_{p}$ of the manifold $M$ itself if and only if the group $\Gamma$ of deck transormations splits accordingly. This means that $\Gamma$ has a set of generators each of which acts only on one of the factors $\tilde{M}_{i}$. We now show that there is even a set of generators which has this property for all splittings of $M$ at the same time:

Lemma 3. There exists a generating set $\Sigma$ of $\Gamma$ such that for any decomposition $M=M_{1} \times \ldots \times M_{p}$ of $M$, each $\sigma \in \Sigma$ acts only on one factor of the corresponding decompositon $\tilde{M}=\tilde{M}_{1} \times \ldots \times \tilde{M}_{p}$.

Proof. Choose $o \in \tilde{M}$ and let $|\gamma|:=\operatorname{dist}(o, \gamma o)$ for each $\gamma \in \Gamma$. Let $\Sigma=\left\{\sigma_{1}, \sigma_{2}, \ldots\right\}$ be a short generating set in the sense of Gromov [G], i.e. $\sigma_{1}$ is chosen with $\left|\sigma_{1}\right|=$ $\min \{|\gamma| ; \gamma \in \Gamma \backslash\{1\}\}$ and $\sigma_{k}$ inductively with $\left|\sigma_{k}\right|=\min \left\{|\gamma| ; \gamma \in \Gamma \backslash \Gamma_{k-1}\right\}$ where $\Gamma_{k-1}$ denotes the subgroup generated by $\sigma_{1}, \ldots, \sigma_{k-1}$. Each $\sigma_{k} \in \Sigma$ (in fact each $\sigma \in \Gamma)$ can be written as $\sigma_{k}=\gamma_{1} \gamma_{2} \ldots \gamma_{p}$ where $\gamma_{i}$ acts only on $\tilde{M}_{i}$. Hence

$$
\left|\sigma_{k}\right|^{2}=\sum_{i=1}^{p}\left|\gamma_{i}\right|^{2} \geq\left|\gamma_{j}\right|^{2}
$$

for all $j$. In case of a strict inequality we have $\gamma_{j} \in \Gamma_{k-1}$ by the choice of $\sigma_{k}$. But this cannot happen for all $j$ since $\sigma_{k} \notin \Gamma_{k-1}$. Thus there exists $i \in\{1, \ldots, p\}$ with $\left|\sigma_{k}\right|=\left|\gamma_{i}\right|$ and $\left|\gamma_{j}\right|=0$ for all $j \neq i$ which means $\sigma_{k}=\gamma_{i}$.

Proof of the Theorem. By Lemma 1 we may assume that $M$ contains no euclidean factor. Let $M=M_{1} \times \ldots \times M_{p}=M_{1}^{\prime} \times \ldots \times M_{q}^{\prime}$ be two decompositions of $M$ into indecomposable factors. According to Lemma 2 we get a decomposition $\tilde{M}=$ $\prod_{i, j} \tilde{M}_{i j} \times F$. Now, if $\sigma$ is any element of the special generating set of Lemma 3, there exist $i \in\{1, \ldots, p\}$ and $j \in\{1, \ldots, q\}$ such that the leaves $\tilde{M}_{i}(p)$ and $\tilde{M}_{j}^{\prime}(p)$ and hence $\tilde{M}_{i j}(p)$ are $\sigma$-invariant for all $p \in \tilde{M}$. In particular, $\sigma$ and hence $\Gamma$ act trivially on $F$. Since $M$ has no euclidean factor, $F$ must be trivial, i.e. $\tilde{M}=\prod_{i, j} \tilde{M}_{i j}$. Furthermore, $\Gamma$ is generated by elements $\sigma$ which act only on one of the factors $\tilde{M}_{i j}$. Thus, by the remark before Lemma 3 we get a corresponding decomposition $M=\prod_{i, j} M_{i j}$ of $M$ with $M_{i j}(m)=M_{i}(m) \cap M_{j}^{\prime}(m)$ for all $m \in M$. Since the $M_{i}$ and $M_{j}^{\prime}$ are indecomposable, the theorem follows.

\section{REFERENCES}

[dR] G. de Rham: Sur la réductibilité d'un espace de Riemann, Comm. Math. Helv. 26 (1952), 328 - 344 MR 14:584a

[G] M. Gromov: Almost flat manifolds, J. Diff. Geom. 13 (1978), 231 - 241 MR 80h:53041

[KN] S. Kobayashi, K. Nomizu: Foundations of Differential Geometry, vol. 1, Interscience, Wiley, New York 1963 MR 27:2945

[M] R. Maltz: The de Rham product decomposition, J. Diff. Geom. 7 (1972), 161 - 174 MR 48:2930

[P] R. Pantilie: A simple proof of the de Rham decomposition theorem, Bull. Math. Soc. Sc. Math. Roumanie 36 (84) (1992), 341 - 343 MR 95m:53068 
[T] H. Takagi: Notes on the cancellation of Riemannian manifolds, Tôhoku Math. J. 32 (1980), 411 - 417 MR 82g:53048

[U] K. Uesu: Cancellation law for Riemannian direct products, J. Math. Soc. Japan 36 (1984), 53 - 62 MR 85c:53072

Institut fur Mathematik, Universität Augsburg, D-86135 Augsburg, Germany

E-mail address: eschenburg@math.uni-augsburg.de

E-mail address: heintze@math.uni-augsburg.de 